Published in final edited form as:

Addict Behav. 2014 September ; 39(9): 1372-1376. doi:10.1016/j.addbeh.2014.02.013.

\title{
Examination of a Short Version of the UPPS-P Impulsive Behavior Scale
}

\author{
Melissa A. Cyders ${ }^{1}$, Andrew K. Littlefield ${ }^{2}$, Scott Coffey $^{3}$, and Kenny A. Karyadi ${ }^{1}$ \\ ${ }^{1}$ Indiana University Purdue University - Indianapolis, Department of Psychology \\ ${ }^{2}$ Texas Tech University, Department of Psychology \\ ${ }^{3}$ University of Mississippi Medical Center
}

\begin{abstract}
The current study examines a recently developed short version of the UPPS-P Impulsive Behavior Scale. Participants were 251 undergraduate students $(59.3 \%$ male; mean age $=21.16(S D=4.18)$; $72 \%$ Caucasian). The short version generally replicated the internal consistency $(0.74-0.88$ across subscales) and inter-scale correlations of the full UPPS-P. Moreover, the estimated loss of shared variance was small ( $0 \%-6.4 \%$ reductions across subscales) as compared to a $66 \%$ timesavings. Structural equation modeling replicated previously supported factor structures and relationships to external outcomes using the full UPPS-P. The short UPPS-P scale should be considered a valid and reliable alternative to the full UPPS-P.
\end{abstract}

\section{Keywords \\ impulsivity; short form; risky behaviors}

\section{Introduction}

Numerous conceptualizations and measurements of impulsivity (e.g. see Evenden, 1999; Moeller, Barratt, Dougherty, Schmitz, \& Swann, 2001) have resulted in the inconsistent use of the term impulsivity, and have produced varying results regarding the link between impulsivity and risky behaviors (Cyders \& Coskunpinar, 2011; Smith et al., 2007; Whiteside \& Lynam, 2001). To ameliorate these issues, researchers have employed exploratory and confirmatory factor analysis on existing impulsivity measures to identify five distinct facets:

\footnotetext{
(C) 2014 Elsevier Ltd. All rights reserved

Correspondence concerning this paper should be addressed to Kenny Karyadi, Indiana University Purdue University Indianapolis, Department of Psychology, 402 N. Blackford St. LD 124, Indianapolis, IN 46202. kkaryadi@iupui.edu. Phone: (425)770-3022.

Publisher's Disclaimer: This is a PDF file of an unedited manuscript that has been accepted for publication. As a service to our customers we are providing this early version of the manuscript. The manuscript will undergo copyediting, typesetting, and review of the resulting proof before it is published in its final citable form. Please note that during the production process errors may be discovered which could affect the content, and all legal disclaimers that apply to the journal pertain.

Author Disclosure

Conflicts of Interest. All authors declare that they have no conflicts of interest.

Contributors. All authors were involved in all aspects of study design and revisions. Melissa Cyders, Andrew Littlefield, and Kenny Karyadi were directly involved in data analyses, data collection, literature review, and manuscript writing.
} 
sensation seeking, lack of premeditation, lack of perseverance, negative urgency, and positive urgency (Cyders \& Smith, 2007; Whiteside \& Lynam, 2001). These five impulsivity facets are assessed using the UPPS-P Impulsive Behavior Scale (UPPS-P; Lynam, Smith, Whiteside, \& Cyders, 2006).

Facets from the UPPS-P have been found account for different types and aspects of risky behaviors (Coskunpinar, Dir, \& Cyders, 2013; Dir, Karyadi, \& Cyders, 2013; Fischer, Smith, \& Cyders, 2008; Glenn \& Klonsky, 2010; Smith et al., 2007). However, due to its length (59 items), completing the UPPS-P can be time consuming (approximately 15 minutes). Researchers often choose briefer measures of "impulsivity" that may lump several distinct impulsivity facets into one overall measure, which can obscure more specific relationships (see Smith, McCarthy, \& Zapolski, 2009). Given the length of the UPPS-P, some researchers might also assess only some of the five impulsivity facets, but this approach decreases the utility of a given dataset.

Lynam (2013) developed a short-form version of the UPPS-P (SUPPS-P). In developing the SUPPS-P, over 1,200 undergraduate students completed the UPPS-P across multiple studies. The goal was to generate four items per subscale (for a total of 20 items on the scale) that would preserve the content coverage and internal consistency coefficients of the original UPPS-P. Development of the short form began with the item with the highest corrected item-total correlation on the subscale. Redundant items, which are items with inter-item correlations greater than 0.50 with the already selected item, were deleted. This process was repeated for the final three items per subscale.

Although this brief measure may dramatically decrease participant burden and thus increase the overall utility of this assessment, no studies have systematically examined the performance of the SUPPS-P. Thus, the goal of the current study was to examine the performance of the short UPPS-P. More specifically, based on the Smith, McCarthy, and Anderson (2000) guidelines for developing valid and reliable alternative short-form measures, we sought to (1) examine the SUPPS-P subscales inter-scale correlations, (2) replicate the factor structure of the full UPPS-P, (3) demonstrate the reliability of the SUPPS-P subscales, (4) demonstrate overlapping variance between short and full UPPS-P subscales, (5) demonstrate meaningful savings in time with minimal loss in shared variance, and (6) analyze the criterionrelated associations with risk-taking behaviors.

\section{Materials and Methods}

\subsection{Participants}

A total of 251 undergraduate students at a Midwestern university (59.3\% male; mean age = $21.16(S D=4.18) ; 72 \%$ Caucasian, 9\% African American, 8\% Hispanic, 6\% Asian, and 4\% Other) completed the Short UPPS-P and a series of outcome questionnaires via an online survey in exchange for credit toward a class research requirement. The study was IRB approved. 


\subsection{Measures}

Demographic measurements included participant age, sex, and ethnicity. The Short UPPS-P Impulsive Behavior Scale (SUPPS-P; Lynam, 2013) was used to measure impulsivity facets. The Alcohol Use Disorder Identification Test (Babor, Higgins-Biddle, Saunders, \& Monteiro, 2001) measured problematic patterns of alcohol use. The National Survey on Drug Use and Health Instrument-Modified (Snodgrass \& LeBaron, 2008) was used to measure the frequency of non-alcoholic substance use in the past 30 days. The Eating Disorder Diagnostic Scale (Stice, Telch, \& Rizvi, 2000) assessed three aspects of problematic eating behaviors: weight and shape concerns, binge eating, and fasting and purging. The South Oaks Gambling Screen (Lesieur \& Blume, 1987) was used to measure gambling behaviors and frequency. The Sexual Behavior Scale (Metzler, Noell, \& Biglan, 1992) was used to assess sexual behaviors. The Self-Harm Inventory (Sansone, Wiederman, \& Sansone, 1998) was used to measure the frequency and presence of self-harm behaviors. The Short UPPS-P items and descriptive statistics for the current sample are presented in Table 1.

\section{Results}

\subsection{Covariance, Validity, and Time Savings Estimates}

Using equation 2 in Smith et al. (2000), SUPPS-P subscales were estimated to be strongly correlated with UPPS-P subscales: negative urgency $(r=0.69)$, positive urgency $(r=0.83)$, lack of perseverance $(r=0.63)$, lack of premeditation $(r=0.71)$, and sensation seeking $(r=$ 0.64). Using equation 3 in Smith et al. (2000), there was an estimated loss in shared variance of $5.4 \%$ for negative urgency, $3.1 \%$ for positive urgency, $0.6 \%$ for lack of perseverance, $0 \%$ for lack of premeditation, and $6.4 \%$ for sensation seeking. Finally, assuming that each item takes 10 to 15 seconds to complete, we are saving approximately 6.5 to 9.75 minutes in administration time (about 66\% in time saved) when SUPPS-P is administered in place of the UPPS-P.

\subsection{Correlations}

We examined the relationships among predictors and outcomes using bivariate correlations. Negative urgency was: (1) weakly and positively correlated with lack of premeditation $(r=$ $0.25, p<0.001)$; (2) moderately and positively correlated with positive urgency $(r=0.42, p$ $<0.001$ ); (3) weakly and positively correlated with problematic alcohol use, weight and shape concerns, and self-harm behaviors $(r=0.22$ to 0.29 , all $p<0.001)$; and (4) moderately and positively correlated with binge eating $(r=0.33, p<0.001)$. Lack of perseverance was: (1) moderately and positively correlated with lack of premeditation $(r=0.48, p<0.001)$; (2) weakly and positively correlated with positive urgency $(r=0.29, p<0.001)$; and (3) weakly and positively correlated with pathological gambling $(r=0.24, p<0.001)$. Lack of premeditation was: (1) weakly and positively correlated with positive urgency $(r=0.24, p<$ 0.001 ); and (2) weakly and positively correlated with problematic drinking, self-harm behaviors, and risky sexual behaviors ( $r=0.13$ to 0.19 , all $p<0.05$ ). Sensation seeking was: (1) weakly and positively correlated with positive urgency $(r=0.30, p<0.001)$; (2) weakly and positively correlated with problematic alcohol use, drug use, and self-harm behavior ( $r=$ 0.14 to 0.26 , all $p<0.05$ ); and (3) weakly and negatively correlated with weight shape and 
concerns $(r=-0.16, p<0.05)$. Positive urgency was weakly and positively correlated with problematic alcohol use, drug use, binge eating, and pathological gambling $(r=0.14$ to 0.23 , all $p<0.05$ ).

\subsection{Confirmatory Factor Analyses}

We examined a series of structural confirmatory factor analyses (as previously done on the full UPPS-P by Cyders \& Smith, 2007) on the SUPPS-P using MPLUS 5.21 (Muthén \& Muthén, 2010) with weighted least squared mean variance (WLSMV) method. First, we tested a one-factor model (Model A), wherein all 20 SUPPS-P items loaded onto a single "impulsivity" factor. This model fit the data poorly: RMSEA $=0.26, \mathrm{CFI}=0.62, \mathrm{TLI}=$ 0.64 , and $\chi^{2}=721.67(d f=42, p<0.001)$. Second, we tested a five-factor model (Model B), wherein we identified five latent traits corresponding to the five SUPPS-P subscales, each with four single item indicators. This model (see top panel of Figure 1) provided a good fit to the data: $\mathrm{RMSEA}=0.07, \mathrm{CFI}=0.97$, TLI $=0.98$, and $\chi^{2}=119.15(d f=57, p<0.001)$. Third, we examined this five-factor model again, adding in a latent overaching trait representing "impulsivity" (Model C). This model provided a worse model fit: RMSEA = $0.11, \mathrm{CFI}=0.93, \mathrm{TLI}=0.94, \chi^{2}=173.13(d f=45, p<0.001)$, and $\chi^{2}$ difference test $=53.98$ $(d f=12 ; p<0.001)$. Finally, we tested a three factor hierarchical model (Model D), wherein three higher order latent variables were identified: emotion-based rash action (negative and positive urgency as lower order factors), sensation seeking, and deficits in conscientiousness (lack of perseverance and premeditation as lower order factors). This model (see bottom panel of Figure 1) provided good fit to the data: $\mathrm{RMSEA}=0.07, \mathrm{CFI}=0.96, \mathrm{TLI}=0.97$, and $\chi^{2}=122.24(d f=53, p<0.001)$. Models D and B were not significantly different from each other in terms of model fit: $\chi^{2}$ difference test $=3.09(d f=4 ; p=0.54)$.

\subsection{Causal Path Model}

We then estimated a structural path model, wherein the individual five SUPPS-P factors were each represented as latent variables with effects on multiple risky behaviors, adjusting for age and sex. The model fit the data reasonably well but was not ideal: RMSEA $=0.09$, $\mathrm{CFI}=0.92$, TLI $=0.93$, and $\chi^{2}=115.90(d f=60, p<0.001)$. With some exceptions, findings generally replicate previous work with the full UPPS-P scale. Negative urgency was significantly associated with alcohol use ( $b=2.38, p<0.05)$, binge eating $(b=2.48, p<$ $0.001)$, and gambling $(b=1.58, p<0.05)$. Lack of perseverance was associated with binge eating $(b=1.73, p<0.05)$. Lack of premeditation was associated with drug use $(b=0.59, p$ $<0.05)$. Sensation seeking was associated with alcohol use $(b=2.43, p<0.05)$, drug use $(b$ $=0.59, p<0.05)$, gambling $(b=1.01, p<0.05)$, and risky sexual behaviors $(b=1.66, p<$ $0.05)$. None of the SUPPS-P traits were associated with self-harm. Positive urgency was unrelated to any of the risk outcomes in the model.

\section{Discussion}

Findings of the study conform to the Smith et al. (2000) guidelines for developing a valid and reliable short-form measure: (1) each scale of the SUPPS-P had adequate reliability; (2) SUPPS-P inter-correlations were generally comparable to UPPS-P inter-correlations (e.g., Cyders \& Smith, 2007); (3) SUPPS-P and UPPS-P scales were estimated to have 
overlapping variance; (4) SUPPS-P administration was associated with adequate time savings and minimal loss in shared variance; and (5) consistent with UPPS-P factor structures previously identified (i.e., Cyders \& Smith, 2007), both a five-factor model and a three-factor hierarchical model fit the data well, whereas a one-factor solution showed poor fit to the data.

Relations between SUPPS-P facets and various external criteria were generally similar to those found using the UPPS-P. Correlates between SUPPS-P facets and alcohol involvement (as assessed by the AUDIT) were similar in magnitude to one non-clinical study (Adams, Ataya, Attwood, \& Munafo, 2012) but were smaller in magnitude compared to another study (Murphy \& MacKillop, 2012). Sensation seeking correlated similarly with drug use compared to a work that utilized the full scale, but other facets from the abbreviated scale showed lower magnitude correlations (Zapolski, Cyders, \& Smith, 2009). Consistent with previous findings (Fischer et al., 2008), negative urgency had the highest magnitude correlations with shape-weight concerns and binge eating.

Consistent with prior studies (Cyders et al., 2007), positive urgency was significantly related to levels of gambling, but this relation was weak in comparison $(r=0.14)$. In contrast with previous findings (Cyders et al., 2007), lack of perseverance was most strongly correlated with levels of gambling $(r=0.24)$. Correlations of SUPPS-P facets and self-harm were largely consistent with previous findings (Dir et al., 2013), with SUPPS-P facets having higher magnitude correlations. Finally, inconsistent with previous findings, positive and negative urgency from the SUPPS-P were weakly and non-significantly correlated with risky sexual behaviors (Zapolski et al., 2009). Findings from the path models were generally consistent with previous findings, except in the case of positive urgency.

Notably, the present study used a primarily Caucasian college-student sample and has a cross-sectional design. Future work should examine the performance and reliability of the SUPPS-P in more diverse samples and longitudinal examination of the SUPPS-P traits would help bolster confidence in the stability of the short form-measured traits. Future work should also examine comparisons between the SUPPS-P and the UPPS-P in the same sample rather than rely on estimates. Despite these limitations, our findings indicate that the SUPPS-P is a viable shortform alternative to the UPPS-P in non-clinical adult samples.

\section{Acknowledgments}

Role of Funding Sources. This research was supported by a National Institute on Alcohol Abuse and Alcoholism Mentored Career Development Award, K01AA020102, to Melissa A. Cyders and a National Institute on Alcohol Abuse and Alcoholism training grant fellowship, T32 AA07462, to Kenny A. Karyadi. These funding sources had no direct involvement with data collection and analyses, manuscript writing, study design, and publication decisions.

\section{References}

Adams S, Ataya AF, Attwood AS, Munafò MR. Effects of alcohol on disinhibition towards alcoholrelated cues. Drug and Alcohol Dependence. 2013; 127:137-142. [PubMed: 22841455]

Babor, TF.; Higgins-Biddle, JC.; Saunders, JB.; Monteiro, MG. The alcohol use disorders identification test: Guidelines for use in primary health care. Geneva, Switzerland: World Health Organization; 2001. 
Coskunpinar A, Dir AL, Cyders MA. Multidimensionality in Impulsivity and Alcohol Use: A MetaAnalysis Using the UPPS Model of Impulsivity. Alcoholism: Clinical and Experimental Research. 2013

Cyders MA, Coskunpinar A. Measurement of constructs using self-report and behavioral lab tasks: Is there overlap in nomothetic span and construct representation for impulsivity? Clinical Psychology Review. 2011; 31:965-982. [PubMed: 21733491]

Cyders MA, Smith GT. Mood-based rash action and its components: Positive and negative urgency. Personality and Individual Differences. 2007; 43(4):839-850.

Cyders MA, Smith GT, Spillane NS, Fischer S, Annus AM, Peterson C. Integration of impulsivity and positive mood to predict risky behavior: development and validation of a measure of positive urgency. Psychological Assessment. 2007; 19:107-118. [PubMed: 17371126]

Dir AL, Cyders MA, Coskunpinar A. From the bar to the bed via mobile phone: A first test of the role of problematic alcohol use, sexting, and impulsivity-related traits in sexual hookups. Computers in Human Behavior. 2013; 29:1664-1670.

Evenden JL. Varieties of impulsivity. Psychopharmacology. 1999; 146:348-361. [PubMed: 10550486]

Fischer S, Smith GT, Cyders MA. Another look at impulsivity: A meta-analytic review comparing specific dispositions to rash action in their relationship to bulimic symptoms. Clinical Psychology Review. 2008; 28:1413-1425. [PubMed: 18848741]

Glenn CR, Klonsky ED. A Multimethod analysis of impulsivity in nonsuicidal self-injury. Personality Disorders: Theory, Research, and Treatment. 2010; 1:67-75.

Lesieur HR, Blume SB. The South Oaks Gambling Screen (SOGS): A new instrument for the identification of pathological gamblers. The American Journal of Psychiatry. 1987; 144:11841188. [PubMed: 3631315]

Lynam, DR. Development of a short form of the UPPS-P Impulsive Behavior Scale. Unpublished Technical Report. 2013.

Lynam, DR.; Smith, GT.; Whiteside, SP.; Cyders, MA. The UPPS-P: Assessing five personality pathways to impulsive behavior (Technical Report). West Lafayette: Purdue University; 2006.

Metzler CW, Noell J, Biglan A. The validation of a construct of high-risk sexual behavior in heterosexual adolescents. Journal of Adolescent Research. 1992; 7:233-249.

Moeller FG, Barratt ES, Dougherty DM, Schmitz JM, Swann AC. Psychiatric aspects of impulsivity. American Journal of Psychiatry. 2001; 158:1783-1793. [PubMed: 11691682]

Murphy C, MacKillop J. Living in the here and now: interrelationships between impulsivity, mindfulness, and alcohol misuse. Psychopharmacology. 2012; 219:527-536. [PubMed: 22169883]

Muthén, LK.; Muthén, B. Mplus 6.0. Los Angeles, CA: Muthén \& Muthén; 2010.

Sansone RA, Wiederman MW, Sansone LA. The Self-Harm Inventory (SHI): Development of a scale for identifying self-destructive behaviors and borderline personality disorder. Journal of Clinical Psychology. 1998; 54:973-983. [PubMed: 9811134]

Smith GT, Fischer S, Cyders MA, Annus AM, Spillane NS, McCarthy DM. On the validity and utility of discriminating among impulsivity-like traits. Assessment. 2007; 14:155-170. [PubMed: 17504888]

Smith GT, McCarthy DM, Anderson KG. On the sins of short-form development. Psychological Assessment. 2000; 12:102-111. [PubMed: 10752369]

Smith GT, McCarthy DM, Zapolski TC. On the value of homogeneous constructs for construct validation, theory testing, and the description of psychopathology. Psychological Assessment. 2009; 21:272-284. [PubMed: 19719340]

Snodgrass, J.; LeBaron, P. National Survey on Drug Use and Health: CAI specifications for programming English version. Rockville, Maryland: Substance Abuse and Mental Health Services Administration; 2008.

Stice E, Telch CF, Rizvi SL. Development and validation of the eating disorder diagnostic scale: a brief self-report measure of anorexia, bulimia, and binge-eating disorder. Psychological Assessment. 2000; 12:123-131. [PubMed: 10887758]

Whiteside SP, Lynam DR. The five factor model and impulsivity: Using a structural model of personality to understand impulsivity. Personality and Individual Differences. 2001; 30:669-689. 
Zapolski TC, Cyders MA, Smith GT. Positive urgency predicts illegal drug use and risky sexual behavior. Psychology of Addictive Behaviors. 2009; 23:348-354. [PubMed: 19586152] 


\section{Highlights}

- SUPPS-P subscales have adequate internal consistency coefficients.

- $\quad$ SUPPS-P subscales inter-correlations are similar compared to UPPS-P subscales.

- $\quad$ SUPPS-P subscales have criterion-related associations with risky behaviors.

- SUPPS-P has a similar factor structure compared to the original UPPS-P.

- Minimal loss in shared variance and significant time savings with SUPPS-P. 

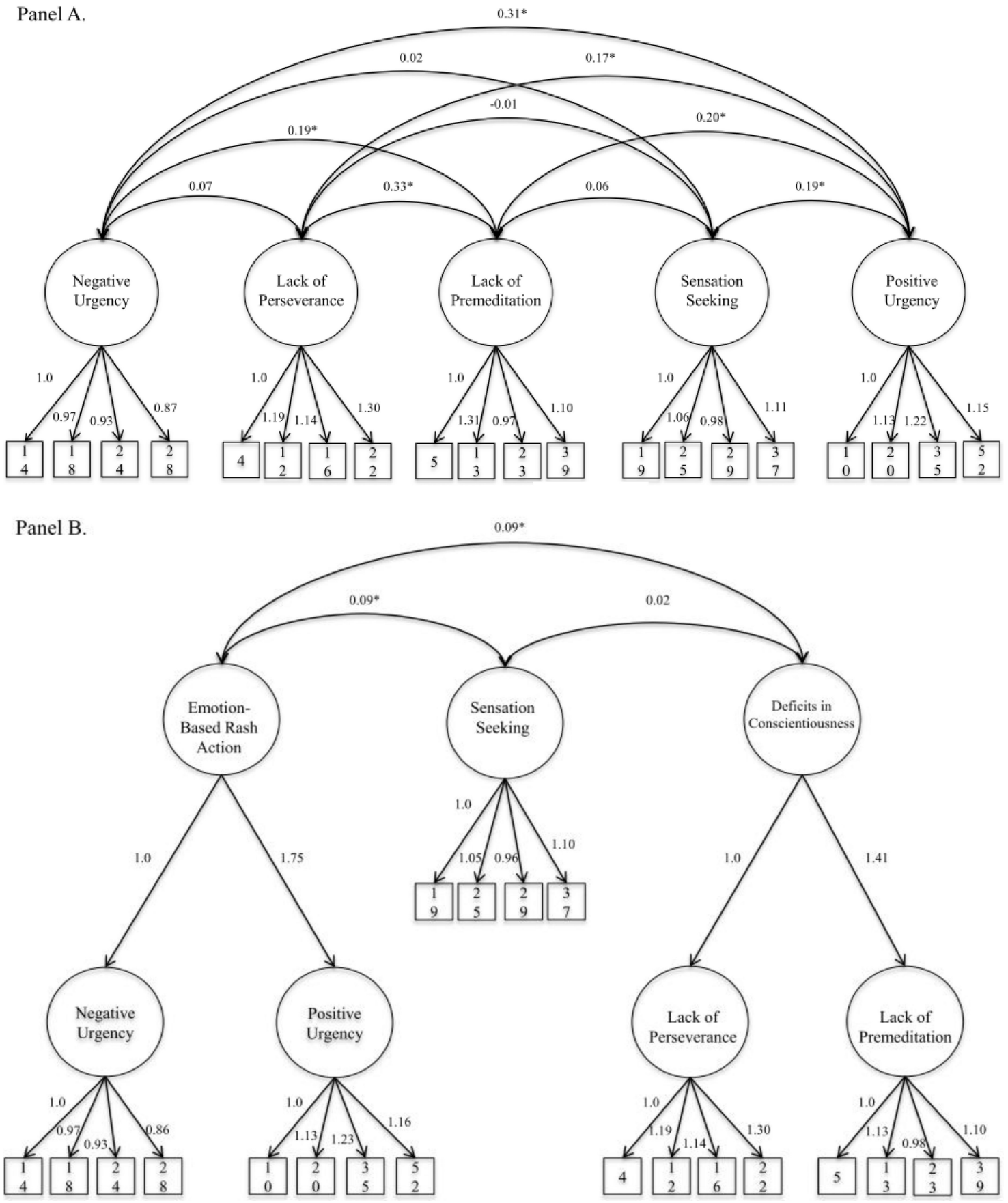

Figure 1.

Five-factor (Panel A) and three factor (Panel B) models of the Short UPPS-P (SUPPSP), with individual SUPPS-P items identified as single item indicators of underlying factors and indicated with item numbers.

Note. $* p<0.01$. 


\section{Table 1}

Final Items Included in the SUPPS-P (Lynam, 2013)

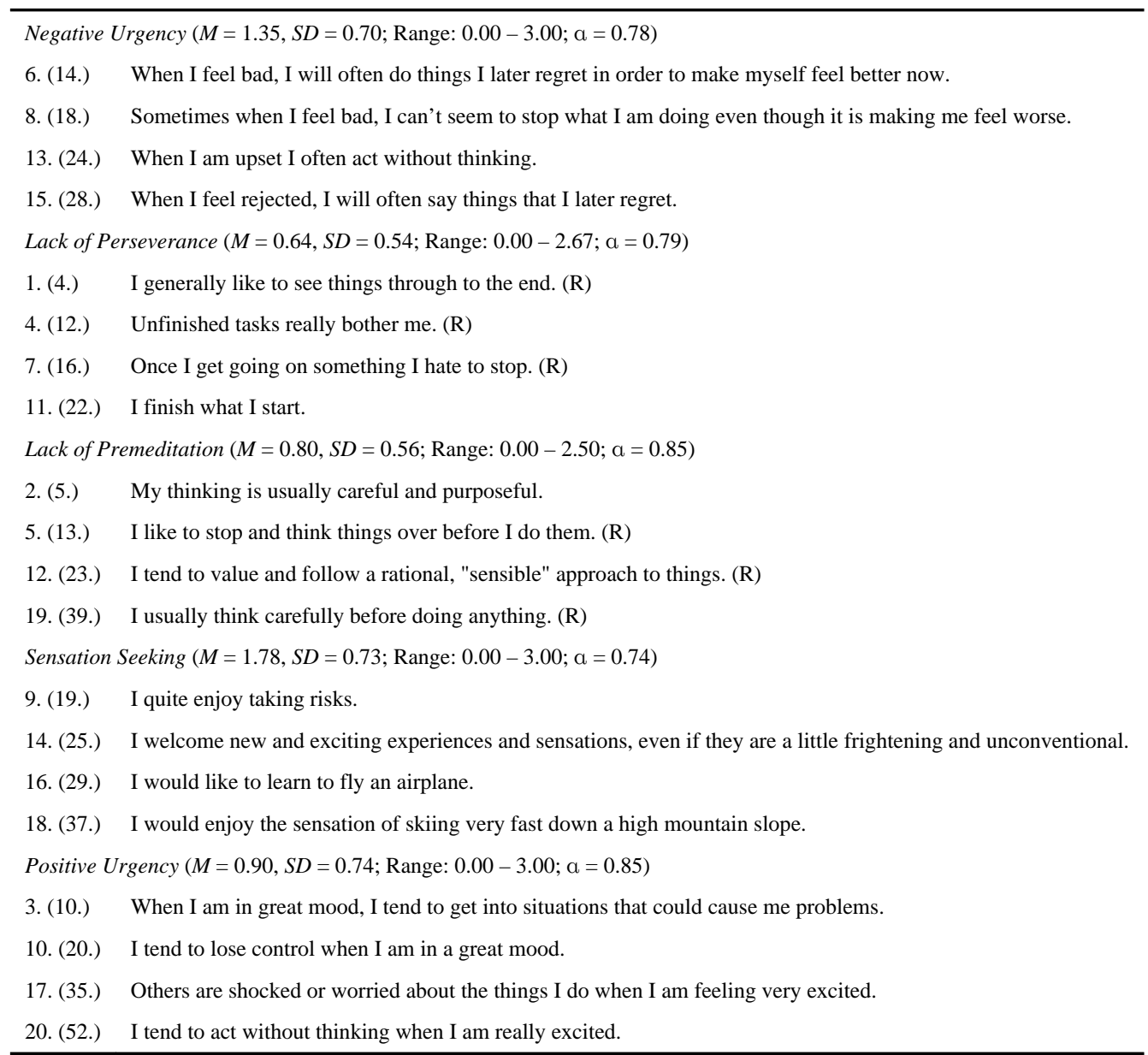

Note. Item numbers indicate the item order on the Short UPPS-P, whereas numbers in parentheses indicate the original item numbers on the UPPSP. All items are rated on a four point scale from 1 (strongly agree) to 4 (strongly disagree). Items with an (R) are reverse coded, so that higher values indicate more impulsive behavior. Total subscale or Mean subscale scores can be calculated. 